\title{
On the centrality of some graphs
}

\author{
Vecdi Aytac \\ Department of Computer Engineering, Faculty of Engineering, Ege University, İzmir, Turkey
}

Received: 15 February 2017, Accepted: 4 August 2017

Published online: 17 October 2017.

\begin{abstract}
A central issue in the analysis of complex networks is the assessment of their stability and vulnerability. A variety of measures have been proposed in the literature to quantify the stability of networks and a number of graph-theoretic parameters have been used to derive formulas for calculating network reliability. Different measures for graph vulnerability have been introduced so far to study different aspects of the graph behavior after removal of vertices or links such as connectivity, toughness, scattering number, binding number, residual closeness and integrity. In this paper, we consider betweenness centrality of a graph. Betweenness centrality of a vertex of a graph is portion of the shortest paths all pairs of vertices passing through a given vertex. In this paper, we obtain exact values for betweenness centrality for some wheel related graphs namely gear, helm, sunflower and friendship graphs.
\end{abstract}

Keywords: Network vulnerability, Network design and communication, Stability, Betweenness centrality of a graph, Gear graph, Helm graph, Sunflower graph and Friendship graph.

\section{Introduction}

Vulnerability is an important concept in network analysis. The vulnerability of a communication network is defined as the measurement of the global strength of its underlying graph. The design of a good communication network must take into account notions such as reliability and vulnerability. When the network requirements are expressed in terms of graph theoretical parameters, the problem of analysis and design of networks becomes finding a graph $G$ satisfying some specified requirement.

Communication systems are often subjected to failures and attacks. A variety of measures have been proposed in the literature to quantify the robustness of networks and a number of graph-theoretic parameters have been used to derive formulas for calculating network reliability. For instance, connectivity of a graph is an important and the earliest measure of robustness of a network [9]. However, the connectivity only partly reflects the ability of graphs to retain connectedness after vertex or edge deletion. Other improved measures were introduced and studied, such as toughness, scattering number, integrity, residual closeness, tenacity etc. [2,3,4,8,9,15,16,17]. In this paper we study the concept of betweenness centrality, introduced as a useful measure of graph vulnerability. The betweenness centrality can be defined as the portion of shortest paths of a graph that pass through a selected vertex. The index attracts the attention due to not only the use in real network analysis but also its mathematical properties [14].

The betweenness centrality of a graph measures the robustness of network under random vertex or edge removals. Removing a vertex or an edge with large betweenness centrality means that the communication between many vertex pairs will be affected, since they will now be forced to exchange information through alternative, possibly longer paths. Graphs that have large betweenness centralities are sensitive to random removal of a set of vertices or edges. The vertex or edge betweenness centrality of a graph does not yield any information about the centralities of different vertices or 
edges, that may have large variation among each other. For networks having the same betweenness centrality, large variations between vertices or edges yield a sensitivity to targeted attacks, since removing the most central vertices may significantly disrupt the functioning in network $[6,13,14]$. It is important that betweenness centrality of the network is only dependent on its size indirectly, through the average distance of the graph. For a fixed order, the average betweenness centrality of a network decreases by adding new edges.

The remainder of this paper is structured as follows. In Section 2, definition and known results for betweenness centrality are given. In section 3, 4, 5, 6 and 7, betweenness centralities of wheel, friendship, helm, gear and sunflower are, respectively, determined and exact values are given. Conclusion is addressed in Section 8.

\section{Betweenness Centrality}

In this paper, we consider simple finite undirected graph that has no self-loops and no more than one edge between any two different vertices. Given a connected graph $G=(V, E), n$ and $m$ denote the number of its vertices and edges, respectively; for $u, v \in V, u \neq v,(u, v)$ denotes an ordered pair of vertices. The set of all neighbors of a vertex $x$ is $N(x)$, and the degree of $x$ is $d(x)=|N(x)|$. We denote by $\delta$ and $\Delta$ the minimum and the maximum degree of the vertices of the graph, respectively $[5,7]$.

For any two vertices $u, v \in V, d(u, v)$ stands for their distance (that is, the length of a shortest $u$-v-path) in $G$. By $d_{u, v}$, we denote the number of all shortest $u$-v-paths, and by $d_{u, v}(x)$ the number of all shortest $u$ - $v$-paths passing through a vertex $x \neq u, v$. Let $b_{u, v}(x)=d_{u, v}(x) / d_{u, v}$, then the betweenness $B(x)$ of a vertex $x$ is defined as $\sum_{(u, v) \in V^{2}} b_{u, v}(x)$ (note that, if $u$ and $v$ belong to different components of $G$, then the corresponding term is not included in the sum). The betweenness of the graph $G$ is $\bar{B}(G)=\frac{1}{n} \sum_{x \in V} B(x)[6,13,14]$.

Theorem 1. [14] $\bar{B}(G)=(n-1)(\bar{l}-1)$, where $\bar{l}$ is mean distance.

Theorem 2. [13] $B(x) \leq(n-1)(n-2)$ for every vertex $x$ of a graph $G$, and the equality holds if and only if $x$ is the center vertex of the star $K_{1, n}$.

Theorem 3. [14] Let $G$ be a graph of order $n$ and let $G^{\prime}$ be the graph obtained from $G$ by connecting two vertices $u, v \in V(G)$ at distance $d=d(u, v)>1$ with a new edge. Then

$$
\bar{B}\left(G^{\prime}\right) \leq \bar{B}(G)-\frac{2(d-1)}{n}
$$

Theorem 4. [14] Let $G^{\prime}=\left(V, E^{\prime}\right)$ be a spanning subgraph of the graph $G=(V, E)$. If $r=\left|E \backslash E^{\prime}\right|$ then

$$
\bar{B}(G) \leq \bar{B}\left(G^{\prime}\right)-\frac{2 r}{n}
$$

Theorem 5. [14] Let $G=(V, E)$ be a graph of order $n$ and size $m$, and let $T$ be a spanning tree of $G$. Then

$$
\bar{B}(G) \leq \bar{B}(T)-\frac{2(m-n+1)}{n}
$$

\section{Betweenness centrality of wheel graph}

A wheel $W_{n}$ of order $n$ is a graph that contains an outer cycle of order $n-1$, and for which every vertex in the cycle is connected to one vertex (which is known as the hub) [12]. The edges of a wheel which include the hub are called spokes. 
In other words, a wheel graph $W_{n}$ is obtained when a additional vertex $c$ not on $C_{n-1}$ is joined to each of the $n-1$ vertices in $C_{n-1}$ by new edges. Let the central vertex of a graph $W_{n}$ be $c$. The central vertex $c$ has a vertex degree $n-1[2,5,7]$.

Theorem 6. If $W_{n}$ is a wheel for $n \geq 7$, then the betweenness value for the wheel graph $W_{n}$ with $n$ vertices is $\bar{B}\left(W_{n}\right)=$ $\frac{(n-4)(n-1)}{2 n}$.

Proof. In the wheel graph $W_{n}$, the central vertex $c$ is adjacent to each vertex of the cycle $C_{n-1}$. We consider the all pair of vertices on $C_{n-1}$. Let $(u, v)$ be on $C_{n-1}$ any pair of adjacent vertices. Since $d(u, v)=1$, there is not any shortest path passing through any vertex except that $u, v$ in $W_{n}$. We have two cases depending on the distance between any two vertices in $C_{n-1}$.

Case 1 Let take to vertex pairs, whose distance over cycle is $d(u, v)=2$. There are $u x v$ and $u c v$ paths between these vertex pairs, where $x \neq u, v$. It is easy to see that $b_{u, v}(x)=1 / 2, x=\overline{1, n}$ and $b_{u, v}(c)=1 / 2$.

While there are only one vertex pairs including $x$ vertex in this path, there are $n-1$ different vertex pairs including the center vertex $c$. Hence, we get

$$
B(x)=1 / 2 \text { and } B(c)=(n-1)(1 / 2)=(n-1) / 2 .
$$

Case 2 Let take to vertex pairs whose distance over cycle is $d(u, v)>2$. There is only $u c v$ path between these vertex pairs. Obviously we have $b_{u, v}(c)=1$. There are $(n-1)(n-6) / 2$ different vertex pairs including the center vertex $c$. Therefore, we get

$$
B(c)=(n-1)(n-6) / 2 .
$$

By summing Case 1 and 2, the betweenness values of each vertex are

$$
B(x)=1 / 2 \text { and } B(c)=(n-1) / 2+(n-1)(n-6) / 2=(n-5)(n-1) / 2 .
$$

Consequently, the betweenness centrality of $W_{n}$ is

$$
\bar{B}\left(W_{n}\right)=\frac{1}{n}\left(\frac{n-1}{2}+\frac{(n-5)(n-1)}{2}\right)=\frac{(n-4)(n-1)}{2 n} .
$$

Thus, the proof is completed.

\section{Betweenness centrality of friendship graph}

The friendship graph $f_{n}$ can be constructed by joining $n$ copies of the cycle graph $C_{3}$ with a common vertex. Another way of obtaining friendship graph is addition of $K_{1}$ and $n$ copies of $K_{2}$ [2,10,11]. Let the central vertex of a friendship graph $f_{n}$ be $c$. The central vertex $c$ has a vertex degree of $2 n$.

Theorem 7. If $f_{n}$ is a friendship graph, then the betweenness value for the friendship graph $f_{n}$ with $2 n+1$ vertices is $\bar{B}\left(f_{n}\right)=\frac{2 n(n-1)}{2 n+1}$.

Proof. The center vertex $c$ in $f_{n}$ is adjacent to each vertices. Because of this, there is not any vertex whose length between the center vertex $c$ is not bigger than 1 . Therefore, we consider only the paths between major vertices. There is only $u c v$ path between the vertex $u$ and the vertex $v, d(u, v)=2$. From the definition of betweenness, we know that $b_{u, v}(c)=1$. There are $(2 n-2) 2 n / 2$ different vertex pairs. Hence, the betweenness value of the center vertex $c$ is

$$
B(c)=(2 n-2) n=2 n^{2}-2 n .
$$


None of the shortest paths that are between all vertex pairs in graph contains any vertex except the center vertex $c$. Hence, the betweenness value of all vertices is zero. Then, $B(x)=0$.

Consequently, the betweenness centrality of the friendship graph $f_{n}$ is

$$
\bar{B}\left(f_{n}\right)=\frac{1}{2 n+1}\left(2 n^{2}-2 n\right)=\frac{2 n(n-1)}{2 n+1} .
$$

Thus, the proof is completed.

\section{Betweenness Centrality Of Helm graph}

A helm graph is denoted by $H_{n}$ obtained by attaching a single edge and vertex to each vertex of the outer circuit of a wheel graph $W_{n}$. The number of the vertices of $H_{n}$ is $2 n-1$ and the number of edges is $3(n-1)$. Let $c$ be the central vertex of $H_{n}$. The central vertex $c$ has a vertex degree of $n$. The vertices of $H_{n} \backslash\{c\}$ are of two kinds: vertices of degree four and one, respectively. The vertices of degree one will be referred to as minor vertices and vertices of degree four to as major vertices $[10,11]$.

Theorem 8. If $H_{n}$ is a helm for $n \geq 6$, then the betweenness value for the helm $H_{n}$ with $2 n-1$ vertices is $\bar{B}\left(H_{n}\right)=$ $\frac{2 n^{3}-6 n^{2}-14 n-7}{2(2 n-1)}$.

Proof. We have four cases depending on the center vertex $c$ and major vertices $k$ of $H_{n}$ :

Case 1 Let examine the paths of between major vertices. Since this case is very similar to the proof of Theorem6, we have

$$
B(k)=1 / 2 \text { and } B(c)=(n-5)(n-1) / 2 .
$$

Case 2 Let examine the paths of between minor vertices in three subcases. Let $x$ and $y$ be different two minor vertices from each other.

Case 2.1 There is only one path between $x$ and $y$ which is $d(x, y)=3$. In this path, there are two different major vertices. Each major vertex $k$ is included only one. From the definition of the betweenness value, we know that $b_{x, y}(k)=1 / 1$ for each major vertex $k$. There are two different vertex pairs including each major vertex. Thus, the betweenness value of the major vertices $k$ is

$$
B(k)=2 .
$$

Case 2.2 There are two different paths between $x$ and $y$ vertices whose distance over cycşle is $d(x, y)=4$. One of these paths is over cycle and the other one is over the center vertex. The center vertex is included only one path. Besides, there are $n$ different vertex pairs. From the definition of the betweenness value, we know that

$$
B(c)=n / 2 \text {. }
$$

The major vertices connected with minor vertices are in both paths. So, we know that $b_{x, y}(k)=2 / 2$. There are two different vertex pairs incliuding each major vertex. The other major vertex is included only one path which is on cycle. Hence, we obtain $b_{x, y}(k)=1 / 2$. By combining obtained two equalities above for each major vertex $k$, we have

$$
B(k)=2(2 / 2)+1 / 2=5 / 2 .
$$


Case 2.3 There is exactly one path over center vertex between vertex pairs whose distance from over cycle is $d(x, y)>4$. There are $n(n-5) / 2$ different vertex pairs including the center vertex. Then, we have

$$
B(c)=n(n-5) / 2 .
$$

There are two major vertex connected with minor vertices in the path. These major vertices are included by $n-5$ vertex pairs. Hence, for each major vertex $k$ we obtain

$$
B(k)=n-5 .
$$

Case 3 The paths between minor and major vertices are examined in three subcases. Let $x$ be a major vertex and $y$ be a minor vertex.

Case 3.1 There is only one path between $x$ and $y$ vertices, $d(x, y)=2$. There is one major vertex between these vertices. Each major vertices are included by two different vertex pairs like this. So we have,

$$
B(k)=2 .
$$

Case 3.2 There are two different paths between $x$ and $y$ vertices, $d(x, y)=3$. While one of them is from over cycle, the other is from over the center vertex. The center vertex is included only one path. Thus, we know that $b_{x, y}(c)=1 / 2$. There are $2 n$ different vertex pairs like this. Therefore, we obtain

$$
B(c)=2 n(1 / 2)=n .
$$

The major vertex which is adjacent with minor vertex is on both paths. So, we know that $b_{x, y}(k)=2 / 2$. There are two different vertex pairs. The other major vertex is included only one in the path which is on cycle. Hence, we obtain $b_{x, y}(k)=1 / 2$. There are also two different vertex pairs for this major vertex. By combining two equalities obtained above for each major vertex $k$, we have

$$
B(k)=2(2 / 2)+2(1 / 2)=3 .
$$

Case 3.3 There is one path between vertex pairs whose distance over cycle is $d(x, y)>3$. The path is over the center vertex. Each major vertex $k$ and the center vertex $c$ are included by $n(n-5)$ vertex pairs. From the definition of betweenness value, we know that

$$
B(k)=B(c)=n(n-5) .
$$

Case 4 There is a two-length path between minor vertices and the center vertex. The path only contains the major vertex. So, we obtain

$$
B(k)=1 .
$$

By summing Case 1,2,3 and 4, the betweenness values of the center vertex $c$ and the major vertices $k$ are respectively,

$$
\begin{gathered}
B(c)=(n-5)(n-1) / 2+n / 2+n(n-5) / 2+n+n(n-5)=\left(4 n^{2}-18 n+5\right) / 2 \\
B(k)=1 / 2+2+5 / 2+(n-5)+2+3+n(n-5)+1=n^{2}-4 n+6 .
\end{gathered}
$$


Consequently, the betweenness centrality for the helm graph $H_{n}$ is

$$
\bar{B}\left(H_{n}\right)=\frac{1}{2 n-1}\left(\frac{4 n^{2}-18 n+5}{2}+(n-1)\left(n^{2}-4 n+6\right)\right)=\frac{2 n^{3}-6 n^{2}-14 n-7}{2(2 n-1)}
$$

Thus, the proof is completed.

\section{Betweenness centrality of gear graph}

Gear graph is a wheel graph with a vertex added between each pair adjacent graph vertices of the outer cycle. Gear graph $G_{n}$ has $2 n+1$ vertices and $3 n$ edges [1,10]. Gear graph $G_{n}$ includes an even cycle $C_{2 n}$. The vertices of $C_{2 n}$ in $G_{n}$ are of two kinds: vertices of degree two and three, respectively. The vertices of degree two will be referred to as minor vertices and vertices of degree three to as major vertices [2,11]. Let the central vertex of gear graph $G_{n}$ be $c$. The central vertex $c$ has a vertex degree of $n$.

Theorem 9. If $G_{2 n}$ is a gear graph for $n \geq 5$, then the betweenness value for the gear graph $G_{2 n}$ with $2 n+1$ vertices is $\bar{B}\left(G_{2 n}\right)=\frac{4 n^{2}-7 n}{2 n+1}$.

Proof. Gear graph has three different vertices, such as the center vertex $c$, major vertex $k$ and minor vertex $m$. The betweenness values of each vertices are examined in four different cases.

Case 1 There are two-length two different paths between the minor vertices and the center vertex. Two paths only contain the major vertex. There are two different vertex pairs like this. So, we have

$$
B(k)=2(1 / 2)=1
$$

Case 2 Let examine the paths between major vertices in two subcases. Let $x$ and $y$ be different two major vertices from each other.

Case 2.1 There are two paths between vertex pairs whose distance from over cycle is $d(x, y)=2$. These paths are $x m y$ over cycle and $x c y$ over the center vertex. It is easy to see that $b_{x, y}(m)=1 / 2$; and $b_{x, y}(c)=, 1 / 2$; While each minor vertex $m$ is included by a vertex pair, the center vertex $c$ is included by $n$ different vertex pairs. Thus, we obtain

$$
B(m)=1 / 2 \text { and } B(c)=n / 2 .
$$

Case 2.2 There is one path between vertex pairs whose distance over cycle is $d(x, y)>2$. This is $x c y$ path over the center vertex. The center vertex $c$ is included by $n(n-3) / 2$ different vertex pairs. From the definition of betweenness value, we know that

$$
B(c)=n(n-3) / 2 .
$$

Case 3 Let examine the paths between minor vertices in three cases. Let $x$ and $y$ be two minor vertices different from each other.

Case 3.1 There is one $x k y$ path between vertex pairs whose distance from over cycle is $d(x, y)=2$. Each major vertex $k$ is included by a vertex pair. From the definition, we have

$$
B(k)=1 \text {. }
$$

Case 3.2 There are five different paths between vertex pairs whose distance over cycle is $d(x, y)=4$. One of them is over cycle and four of them are over the center vertex. Thus, we obtain $b_{x, y}(c)=4 / 5$. The path over 
cycle contains the minor vertex $m$. So, we have $b_{x, y}(m)=1 / 5$ While the center vertex is included by $n$ different vertex pairs, each minor vertex $m$ is included by a vertex pair. Therefore, we obtain

$$
B(c)=4 n / 5 \text { and } B(m)=1 / 5 .
$$

These five paths include the major vertex other than the above studied the center and minor vertex. There are $n$ different vertex pairs providing this condition. For each major vertex $k$ : each major vertex $k$ is contained only four of these vertex pairs. In two of these, the betweenness values are $2 / 5$, in others two are $3 / 5$. By combining obtained two equalities for each major vertex $k$, we obtain

$$
B(k)=2(2 / 5+3 / 5)=2 .
$$

Case 3.3 There are four different paths between vertex pairs whose distance over cycle is $d(x, y)>4$. The center vertex is in all these paths. It is included by $n(n-5) / 2$ different vertex pairs. From the definition, we obtain

$$
B(c)=\frac{(n(n-5) / 2) 4}{4}=n(n-5) / 2 .
$$

These four paths also include the major vertex. The two paths contain the minor vertex. Each minor vertex $k$ is included by $2(n-5)$ different vertex pairs. So, we have

$$
B(k)=2(n-5)(2 / 4)=n-5 .
$$

Case 4 Let examine the paths between $x$ and $y$ in two cases. Let $x$ be a major vertex and $y$ be a minor vertex.

Case 4.1 There are three different paths between vertex pairs whose distance over cycle is $d(x, y)=3$. One of them is over cycle and the other two are over the center vertex. For the center vertex $c$, we know that $b_{x, y}(c)=2 / 3$. There are $2 n$ different vertex pairs providing this condition and including the center vertex. Thus, we have

$$
B(c)=2 n(2 / 3) .
$$

The minor vertex $m$ is only in path over cycle. It is included by 2 different vertex pairs. From the definition, we obtain

$$
B(m)=2(1 / 3)=2 / 3 .
$$

These three paths include the major vertex other than the above studied the center and minor vertex. There are $2 n$ different vertex pairs providing this condition. For each major vertex $k$ : each major vertex $k$ is contained only four of these vertex pairs. In two of these, the betweenness values are $2 / 3$, in others two are $1 / 3$. By combining obtained two equalities for each major vertex $k$, we obtain

$$
B(k)=, 2(2 / 3+1 / 3)=2 \text {. }
$$

Case 4.2 There are two different paths between vertex pairs whose distance over cycle is $d(x, y)>3$. The center vertex $c$ is in all these paths. The major vertex $k$ is in only one path. Hence, in these two paths the betweenness values of the major vertex $k$ and the center vertex $c$ are $1 / 2$ and $2 / 2$, respectively. While each major vertex $k$ is included by $2(n-4)$ different vertex pairs, the center vertex $c$ is included by $n(n-4)$ different vertex pairs. From the definition, we obtain

$$
B(k)=(2(n-4)) 1 / 2=n-4
$$




$$
B(c)=(n(n-4)) 2 / 2=n(n-4)
$$

By summing Case 1,2, 3 and 4, the betweenness values of the center vertex $c$, major vertex $k$ and minor vertex $m$ are, respectively;

$$
\begin{gathered}
B(c)=\frac{n}{2}+\frac{n(n-3)}{2}+\frac{4 n}{5}+\frac{n(n-5)}{2}+\frac{4 n}{3}+n(n-4)=2 n^{2}-\frac{161 n}{30} \\
B(k)=1+1+2+n-5+2+n-4=2 n-3 \\
B(m)=1 / 2+1 / 5+2 / 3=41 / 30 .
\end{gathered}
$$

As a consequence, the betweenness value of gear graph is

$$
\bar{B}\left(G_{2 n}\right)=\frac{1}{2 n+1}\left[2 n^{2}-\frac{161 n}{30}+n(2 n-3)+n \frac{41}{30}\right]=\frac{4 n^{2}-7 n}{2 n+1}
$$

Thus, the proof is completed.

\section{Betweenness centrality of sunflower graph}

Sunflower graph $S F_{n}$ consists of a wheel with central vertex $c$ and an $n$-cycle $v_{0}, v_{1}, v_{2}, \ldots, v_{n-1}$ and additional $n$ vertices $w_{0}, w_{1}, w_{2}, \ldots, w_{n-1}$ where $w_{i}$ is joined by edges to $v_{i}, v_{i+1}$ for $i=0,1,2, \ldots n-1$ where $i+1$ is taken modulo $n$. $S F_{n}$ has order $2 n+1$ and size $4 n$. Let $c$ be the central vertex of $S F_{n}$. The central vertex $c$ has a vertex degree of $n$. The vertices of $S F_{n} \backslash\{\mathrm{c}\}$ are of two kinds: vertices of degree five and two, respectively. The vertices of degree two will be referred to as minor vertices and vertices of degree five to as major vertices [10,11].

Theorem 10. If $S F_{n}$ is a sunflower graph for $n \geq 7$, then the betweenness value for the sunflower graph $S F_{n}$ with $2 n+1$ vertices is $\bar{B}\left(S F_{n}\right)=\frac{8 n^{2}-24 n+5}{2(2 n+1)}$.

Proof. Sunflower graph has three different vertices, including the center vertex $c$, the major vertex $k$ and the minor vertex $m$. None of the shortest paths that are between all pairs of vertices in graph does not passes through minor vertices. Hence, the betweenness value of $m$ minor vertices is zero. The betweenness values of the center vertex $c$ and $k$ major vertices are examined in four different cases.

Case 1 Let examine the paths between major vertices. Since this case is very similar to the proof of Theorem6, we have

$$
B(k)=1 / 2 \text { and } B(c)=(n-5)(n-1) / 2 .
$$

Case 2 Let examine the paths between minor vertices in four cases. Let $x$ and $y$ be minor vertices different from each other.

Case 2.1 There is only one path between vertex pairs whose distance over cycle is $d(x, y)=2$. This path is $x k y$ path. There is only one vertex pair including the major vertex and providing this condition. From the definition, we know that for each major vertex $k$

$$
B(k)=1 \text {. }
$$

Case 2.2 There is only one path between vertex pairs whose distance over cycle is $d(x, y)=3$. The path contains the major vertex. There is two different vertex pairs including the major vertex and providing this condition. 
Thus, we obtain for each major vertex $k$

$$
B(k)=2(1 / 1)=2 .
$$

Case 2.3 There are exactly five different paths between vertex pairs whose distance over cycle is $d(x, y)=4$. One of them is over cycle; other remaining paths are over the center vertex. The center vertex $c$ is included by $n$ different vertex pairs as distance over cycle $d(x, y)=4$. Then, we have

$$
B(c)=n(4 / 5) \text {. }
$$

These five paths include the major vertex other than the above studied the center vertex. For each major vertex $k$ : there are five different vertex pairs providing this condition and also including each major vertex $k$. In two of these, the betweenness values are $3 / 5$, in the other two are $2 / 5$ and in one is $1 / 5$. By combining obtained these equalities for each major vertex $k$, we obtain

$$
B(k)=1 / 5+2(3 / 5+2 / 5)=11 / 5
$$

Case 2.4 There are four different paths between vertex pairs whose distance over cycle is $d(x, y)>4$. The center vertex is included in every four paths. There are $n(n-7) / 2$ different vertex pairs as like this. From the definition, we know that

$$
B(c)=(n(n-7) / 2) 4 / 4=n(n-7) / 2
$$

Two of these paths include the major vertex. Hence, the betweenness value of the major vertex $k$ is $2 / 4$. There are $2(n-7)$ different vertex pairs. So, we obtain for each major vertex $k$

$$
B(k)=2(n-7)(2 / 4)=n-7 .
$$

Case 3 Let examine the paths between minor and major vertices in three cases. Let $x$ be minor and $y$ be major vertices.

Case 3.1 There is only one path between vertex pairs whose distance over cycle is $d(x, y)=2$. This is $x k y$ path. There is two vertex pairs including the major vertex and providing this condition. From the definition, we know that for each major vertex $k$

$$
B(k)=2 .
$$

Case 3.2 There are three different paths between vertex pairs whose distance over cycle is $d(x, y)=3$. One of them is over cycle, other remaining paths are over the center vertex. From the definition, we know that $b_{x, y}(c)=2 / 3$. The center vertex $c$ is included by $2 n$ different vertex pairs as distance over cycle $d(x, y)=$ 4. Then, we have

$$
B(c)=2 n(2 / 3)=4 n / 3 .
$$

In these three paths, there are three major vertices. One of them is included in two different paths and the others are included in only one path. There are a total of six different vertex pairs providing this condition. In two of these, the betweenness values of each major vertex $k$ are $2 / 3$ and in the others is $1 / 3$. This is valid each major vertex $k$. By combining obtained these equalities for each major vertex $k$, we obtain

$$
B(k)=2(2 / 3+1 / 3+1 / 3)=8 / 3
$$


Case 3.3 There are two different paths between vertex pairs whose distance over cycle is $d(x, y)>3$. These paths are over the center vertex. So, the center vertex $c$ is included in both two paths. The center vertex is also included by $n(n-6)$ different vertex pairs as like this. Therefore, we get

$$
B(c)=n(n-6)(2 / 2)=n(n-6)
$$

Only one of these two paths contains the major vertex. For each major vertex $k$, we obtain $b_{x, y}(k)=1 / 2$. There are $2(n-6)$ different vertex pairs providing this condition and also including each major vertex $k$. Then, for each major vertex $k$, we obtain

$$
B(k)=2(n-6)(1 / 2)=n-6
$$

Case 4 There are two different paths between each minor vertices and the center vertex. Each major vertex $k$ is included in only one path. There are 2 different vertex pairs providing this condition. Hence, we get

$$
B(k)=2(1 / 2)=1
$$

By summing, Case 1, 2, 3 and 4, the betweenness values of the center vertex $c$ and major vertex $k$ are, respectively;

$$
\begin{gathered}
B(c)=\frac{(n-5)(n-1)}{2}+\frac{4 n}{5}+\frac{n(n-7)}{2}+\frac{4 n}{3}+n(n-6)=\frac{60 n^{2}-311 n+75}{30} \\
B(k)=1 / 2+1+2+11 / 5+n-7+2+8 / 3+n-6+1=2 n-49 / 30 .
\end{gathered}
$$

As a consequence, the betweenness value of sunflower graph is

$$
\bar{B}\left(S F_{n}\right)=\frac{1}{2 n+1}\left(\frac{60 n^{2}-311 n+75}{30}+n\left(2 n-\frac{49}{30}\right)\right)=\frac{8 n^{2}-24 n+5}{2(2 n+1)} .
$$

Thus, the proof is completed.

\section{Conclusion}

In this paper, we calculate the betweenness centrality of some wheel related networks. Calculation of betweenness centrality for simple graphs is important because the betweenness centrality of more complex graphs can be calculated by using betweenness centrality of its (simple) parts. Very good practical results can be achieved if the betweenness centrality is calculated for some real networks (e.g. the Power grid). This parameter is of particular interest because it is considered to be a reasonable measure for the vulnerability of graphs.

\section{Competing interests}

The authors declare that they have no competing interests.

\section{Authors' contributions}

All authors have contributed to all parts of the article. All authors read and approved the final manuscript. 


\section{References}

[1] A. Brandstädt, V. B. Le, J. P. Spinrad: Graph classes: a survey, Society for Industrial and Applied Mathematics (SIAM), Philadelphia, PA, 1999.

[2] A. Aytaç, and Z. N. Odabaş: Residual Closeness of Wheels and Related Networks, Internat. J. Found. Comput. Sci., 22, 1229-1240, 2011.

[3] C. A. Barefoot, R. Entringer and H. Swart: Vulnerability in graphs-a comparative survey, J. Combin. Math. Combin. Comput., 1, $13-22,1987$.

[4] D. R. Woodall: The binding number of a graph and its Anderson number, Journal of Combinatorial Theory, Series B, 15, 225 255, 1973.

[5] F. Buckley and F. Harary: Distance in Graphs, Addison-Wesley Publishing Company Advanced Book Program, Redwood City, CA, 1990.

[6] F. Comellas, S. Gago: Spectral bounds for the betweenness of a graph, Linear Algebra Appl., 423, 74 - 80, 2007.

[7] G. Chartrand and L. Lesniak: Graphs and Digraphs, Second Edition, Wadsworth \& Brooks/Cole Advanced Books \& Software, Monterey, CA, 1986.

[8] H. A. Jung: On a class of posets and the corresponding comparability graphs, Journal of Combinatorial Theory, Series B, 24 (2), $125-133,1978$.

[9] H. Frank, I. T. Frisch: Analysis and design of survivable networks, IEEE Transactions on Communications Technology, 18(5), 501 - 519, 1970.

[10] I. Javaid, S. Shokat: On the partition dimension of some wheel related graphs, Journal of Prime Research in Mathematics, 4, 154 $164,2008$.

[11] J. A. Gallian: A dynamic survey of graph labeling, Elect. Jour. Combin., 15, DS6, 2008.

[12] M. Arockiaraj, P. Manuel, I. Rajasingh, B. Rajan: Wirelength of 1-fault hamiltonian graphs into wheels and fans Inform. Process. Lett., 111, 921-925, 2011.

[13] L. C. Freeman: A set of measures of centrality based on betweenness, Sociometry, 40, No. 1, 35 - 41, 1977.

[14] S. Gago, J. Hurajová and T. Madaras: Notes on the betweenness centrality of a graph, Math. Slovaca, 62, No. 1, 1-12, 2012.

[15] T. Turaci and M. Ökten : Vulnerability Of Mycielski Graphs Via Residual Closeness, Ars Combinatoria, Volume CXVIII, 419 427, 2015.

[16] V. Aytac and T. Turaci: Computing the closeness Centrality in Some Graphs, Submitted.

[17] Z. N. Odabaş and A. Aytaç: Residual Closeness in Cycles and Related Networks, Fundamenta Informaticae, 124, 297 - $307,2013$. 